\title{
The Intelligibility OBJection Against UNDERDETERMINATION
}

\author{
Rogério Passos SeVero \\ Universidade Federal de Santa Maria
}

\begin{abstract}
One of the objections against the thesis of underdetermination of theories by observations is that it is unintelligible. Any two empirically equivalent theories - so the argument goes - are in principle intertranslatable, hence cannot count as rivals in any non-trivial sense. Against that objection, this paper shows that empirically equivalent theories may contain theoretical sentences that are not intertranslatable. Examples are drawn from a related discussion about incommensurability that shows that theoretical non-intertranslatability is possible.
\end{abstract}

Keywords: Empirical equivalence; underdetermination; incommensurability; untranslatability.

\section{Introduction}

The thesis of underdetermination of theories by observations is notoriously controversial: while some philosophers have set out to prove it, others claim that it is no more than a conjecture, and others still argue that it is untenable. Assent and dissent vary according to how strong the formulation of the thesis is. Laudan and Leplin (1991), for example, conceive the thesis as saying that "every empirically successful theory has empirically equivalent counterparts" (p. 459 - emphasis added). Not surprisingly, given such a broad formulation, they think the thesis "stands refuted" (p. 466). Hoefer and Rosenberg (1994) restrict underdetermination to global theories and claim that while no a priori argument can rule out the possibility of rival empirically equivalent and empirically adequate systems of the world, there remain "formidable" (p. 605) constraints on the realization of that possibility. Finally, Stanford (2001), following Sklar (1975 and 1981), has put forth a milder version of the thesis, dubbed "transient underdetermination", which does not entail the possibility of rival theories that entail the same observable predictions, but merely the possibility of rival theories that are equally warranted by currently available evidence. Transient underdetermination says that "there might be garden-variety alternative hypotheses, not yet even imagined or entertained by us, but nonetheless consistent with or even equally well confirmed by all the actual evidence we happen to have at hand" (2001, p. S7). Transient underdetermination, Stanford argues, is unlike stronger versions of the thesis in that it is well-supported by an inductive argument over the history of science. ${ }^{1}$

Principia 16(1): 121-146 (2012).

Published by NEL — Epistemology and Logic Research Group, Federal University of Santa Catarina (UFSC), Brazil. 
The purpose of this paper is not to argue for or against any particular version of the thesis, but rather to respond to two related objections that say that the notion of empirically equivalent rival theories is unintelligible. This paper focuses on the second of the three versions mentioned above, because on the first formulation the thesis is clearly false and on the third it is so weak that it is hardly distinguishable from holism. ${ }^{2}$ The second, intermediately strong version, was put forth rather hesitantly by Hoefer and Rosenberg (1994) and less hesitantly by Quine (1975a). It says that for any given global theory, an empirically equivalent rival theory is possible. This is often presented as a conjecture. The issue here is whether it makes sense. Can we understand the idea of rival empirically equivalent theories?

Dummett says we cannot:

... there could be nothing to prevent our attributing the apparent incompatibility [of two empirically equivalent theories] to equivocation. Indeed, if we can establish the empirical equivalence of the two theories, we must be able to find translations from each to the other. (1981, p. 617n)

According to Dummett, empirically equivalent theories assert exactly the same in different (intertranslatable) languages, hence they are not rivals in any interesting sense. If they are intertranslatable, then whatever differences they have is merely terminological. No substantial rivalry between then can ever come up. Section 2 below details this objection and responds to it based on writings by Quine on the topic. Section 3 presents and responds to a similar objection derived from the writings of Putnam and Davidson against the thesis of incommensurability. The concluding section says that there is a clear enough sense in which the notion of empirically equivalent rival global theories is conceivable.

\section{Quine, underdetermination, and partial untranslatability}

Quine revised his formulation of the thesis of underdetermination over the years. ${ }^{3}$ His initial formulations (1955, p. 254; 1960, p. 23) merely say that given the lack of a tight fit between theories and observations, one can expect various alternative theories of the world to imply the same observation categoricals, no matter how large the number of observation reports is. ${ }^{4}$ Whether theories conform to all observations, or all possible observations, is inessential to the thesis; what matters is that they entail the same observation categoricals (Quine 1990a, p. 53). Whatever evidence can be summoned in favor of one theory will then likewise corroborate its empirically equivalent alternatives.

In later writings (for example, 1969b, 1970) Quine described underdetermined theories as empirically equivalent and logically incompatible. This was an attempt to characterize more precisely what kind of rivalry empirically equivalent theories 
can be thought to have, but it proved to be an inconsequential move. All logical incompatibilities can be avoided by the "trivial expedient" of changing the spelling of the theoretical terms which occur in the incompatible sentences (see Quine 1992b, pp. 97-8). If one theory contains the sentence " $\forall x) F x$ ", while the rival theory contains the sentence " $(\forall x) F x$ ", then surely $F$ must be a theoretical predicate (given that the two theories agree on all observable predictions). Hence, as a matter of charity of interpretation, occurrences of " $F$ " in each theory are best understood as different predicates. The equivocation can be altogether avoided by replacing all occurrences of " $F$ " in one theory by occurrences of a new predicate, say, " $G$ ". By systematically repeating this expedient for each pair of incompatible sentences among the two theories, logical incompatibility can be avoided. We are now left with two compatible theories which contain distinct but possibly non-intertranslatable sets of predicates.

Accordingly, Quine's final formulation of the thesis merely states that there are global theories which we might somehow manage to recognize as empirically equivalent and yet defy all attempts at intertranslation (1975a, 1981, 1992b), that is, we will fail at rendering them logically equivalent by translating both to a common language. Failure at intertranslation, however, may here stem from our own epistemic shortcomings. Perhaps an intertranslation is always possible, even if we are to remain ignorant of how to achieve it. Hence, Quine's final, "vague and modest" thesis of underdetermination does not rule out the possibility that all empirically equivalent theories might be intertranslatable versions of each other. Instead, it states merely that we may find empirically equivalent systems of the world for which we will find no intertranslation. This, he maintains, is a plausible thesis, supported by the "less-than-rigid" connections that obtain between theoretical hypotheses and observations and by the possibility of scientific developments divergent with our own. Whether or not such possibilities will ever be realized is then an empirical question not to be answered by a definition, even if one cannot be completely clear on what they might entail or require (see Quine 1981, p. 181).

Quine's characterization of the thesis leaves unclear what such alternative scientific developments might look like, and whether we would recognize them as such if we were to encounter whatever theories they might have produced. To be sure, it can hardly be denied that alternative scientific developments are possible. But that alone provides little clue as to what they might entail and how we would understand them, given that they would be partly untranslatable into the language of our own theories. One way of overcoming the difficulty is to provide an illustration of theories that are empirically equivalent but cannot be rendered logically equivalent through translation. Such an illustration, however, would at most be an imperfect case of underdetermination, since a full-fledged case would have to comprise rival systems of the world yet to be devised. Although physics is a discipline which seeks "full 
coverage" and may one day instantiate the idea of a system of the world, as things stand today physics itself is yet to be unified (quantum mechanics and general relativity are still somewhat at odds with each other) and the coverage it provides is less than total. Nevertheless, a partial illustration of the thesis would suffice both to advance our understanding of what it entails and to undermine the objection that all empirically equivalent theories are ipso facto intertranslatable.

Quine was not too prodigal when it came to providing examples of what he meant by empirically equivalent but non-intertranslatable theories. There was one case, however, to which he returned several times over the years. It is due to Poincaré, and part of an argument to the effect that the laws of geometry are not experimentally testable, a thesis to which Quine does not subscribe. Poincaré argues that those laws are imposed by us onto what we perceive:

Beings whose minds were made as ours, and with senses like ours, but without any preliminary education, might receive from a suitably-chosen external world impressions which would lead them to construct a geometry other than that of Euclid, and to localize the phenomena of this external world in a non-Euclidean space, or even in space of four dimensions. As for us, whose education has been made by our actual world, we should have no difficulty in referring phenomena to our Euclidean space. (1952, p. 51)

Poincaré illustrates that possibility with the following thought-experiment:

Suppose (...) a world enclosed in a large sphere and subject to the following laws:-The temperature is not uniform; it is greatest at the centre and gradually decreases as we move towards the circumference of the sphere, where it is absolute zero. The law of this temperature is as follows:-If $R$ be the radius of the sphere, and $r$ the distance of the point considered from the centre, the absolute temperature will be proportional to $R^{2}-r^{2}$. Further, I shall suppose that in this world all bodies have the same co-efficient of dilatation, so that the linear dilatation of any body is proportional to its absolute temperature. Finally, I shall assume that a body transported from one point to another of different temperature is instantaneously in thermal equilibrium with its new environment. (p. 65)

In Poincaré's example, bodies shrink as they move away from the center and become colder. As they move away from the center, however, the proportion between the size of the object and the distance between it and the edges of that space remains constant. If it's half-way to the edge, its size will have shrunk accordingly; if it's $\frac{3}{4}$ of the way to the edge, its size will have shrunk even further, and so forth. As a result, by traveling finite distances (in proportion to the size of its body) in finite amounts of time it will never reach the edges of the sphere. The distance between the position where an object is inside the sphere and the edges of that sphere remains always infinite in proportion to the size of the object. Measured from inside the sphere the 
distance from any point to the edges is infinite, since all measuring instruments will also shrink proportionally as they approach the edges. At the edge, all objects shrink to a point.

Poincaré's example purports to show that observations alone cannot determine the geometrical laws that are true of the space in which we live. In his thoughtexperiment, the laws of both Euclidean geometry and Bolyai-Lobachevskian geometry accord with observations; choice between them is thus underdetermined by observations. For Poincaré, this would show that they are purely conventional and describe not the structure of space, but the properties we choose to assign to space. Quine used Poincaré's examples as an illustration of thesis of underdetermination, but rejected his conclusions about the nature of the laws of geometry. Unlike Poincaré, Quine regarded the laws of geometry as having the same epistemic status as the more abstract and theoretical laws of physics.

The example illustrates what rivalry between empirically equivalent theories might look like, but no more than that. Insofar as the theory of general relativity is true, both Euclidean and Bolyai-Lobachevskian geometries are false of our physical space. Observations thus conflict with both those geometries when each is assessed in conjunction with general relativity. An actual case of empirically equivalent rival theories would require two global theories which predict the same, which is not the case with Poincaré's examples.

Quine picked up the example in the 1970s but changed his mind a few times about its status over the following couple of decades. At first, he conceived it as a bad illustration of underdetermination, because the two rival geometries could be rendered logically equivalent by reconstrual of predicates, or translation. He thought of the two geometries mentioned by Poincaré as similar to the case of a physics theory in which all occurrences of "proton" have been substituted for "electron", and vice-versa. The latter theory and the original one without the substitutions are of course logically incompatible, since they imply contradictory sentences about electrons and protons. For example, one theory might say that protons are positively charged, whereas the other that they are negatively charged. Nevertheless, those two theories are clearly intertranslatable, and can without difficulty be rendered logically equivalent by translating both into the vernacular English of contemporary physics. The two formulations can thus be viewed as versions of the same theory: what one formulation means by "proton" is just what the other means by "electron". The difference between them is purely terminological. By 1975, Quine thought that the Euclidean and Bolyai-Lobachevskian geometries which the example by Poincaré brings up would be likewise intertranslatable, although less easily or straightforwardly so:

[F]ollowing Poincaré, suppose the two theories alike except that one of them

Principia 16(1): 121-146 (2012). 
assumes an infinite space while the other has a finite space in which bodies shrink in proportion to their distance from centre. Even here we want to say that the difference is rather terminological than real; and our reason is that we see how to bring the theories into agreement by translation: by reconstruing the English of one of the theories. (Quine 1975b, p. 80; see also Quine 1975a, p. 322)

No translation was ever provided, however, and later Quine came to accept Poincaré's thought-experiment as containing a good illustration of underdetermination. The notion of 'the center of space' is ambiguous in Euclidean geometry. In fact in any space that is infinite, any point can count as a center. In BolyaiLobachevsky geometry, on the other hand, only one point counts as the center. A complete intertranslation must therefore fail; the two theories cannot be rendered logically equivalent by reconstrual of predicates:

One may ask what to count as distinct theories and what to count merely as different formulations of a single theory. If two empirically equivalent theory formulations are interconvertible sentence by sentence through reinterpretation of terms, surely we should regard them as formulating a single theory. If we just interchange the words 'proton' and 'electron' in a physics treatise, we already have two formulations that are related in that trivial way. On the other hand Poincaré's two theories are not thus interconvertible, since one of them singles out a central point of space while the other provides for no such singularity. (Quine 1990b, p. 13)

In $1992 \mathrm{~b}$, the same point is made again. The two geometries, Quine says, ... differ (...) more deeply than in the mere choice of words. The theory with the finite space makes crucial use of a theoretical term that admits of no counterpart in the theory with the infinite space - namely, 'center of space'. (p. 97)

So here we would have, on Quine's later view, theories that are empirically equivalent but cannot be rendered logically equivalent through translation. Partial intertranslation is of course perfectly possible, and indeed some of the sentences - in particular observation sentences and categoricals — will be logically equivalent. But the notion of 'center of space' of Poincaré's disk geometry and other notions that are directly connected to it have no counterparts in Euclidean geometry. Likewise, the notion of 'finite space' has no counterpart in the Bolyai-Lobachevsky geometry.

It is true that the net empirical implications of the two theories in Poincarés example are indeed the same. Hence, one might conclude, the two, taken as wholes, are translations of one another, even if particular portions or sentences of one have no counterparts in the other. This, according to Quine, would be "a queer translation" (1969a, p. 79). In cases where no translation sentence by sentence is available 
or possible, he wrote, we "might better speak (...) not of translation but simply of observational evidence for theories" (ibid). What we should properly call a translation is something which allows for fluent dialogue and negotiation across languages; hence something that requires sentence by sentence translation, even if not word for word.

Among theories that are empirically equivalent, one expects observation sentences and categoricals to be intertranslatable. Such sentences command immediate assent or dissent on the part of all competent speakers of the language that witness the occasion described. Theories are empirically equivalent if they entail the same observation categoricals, or, in case they are formulated in different languages, if they entail intertranslatable observation categoricals. Perhaps some or even most of the more theoretical statements are likewise intertranslatable. But if the two theories are to be thought of as genuinely distinct, rival theories, then there must be at least one sentence or a set of interconnected sentences which cannot be intertranslated. The two theories must be such that no reconstrual of predicates or translation can render them logically equivalent.

Translatability is a relation between two or more languages. In Poincaré's example, all sentences which are true of the Euclidean sphere and contain the phrase 'the center of space' have no counterpart in Bolyai-Lobachevsky geometry. The notion of a center can be defined for an Euclidean sphere $S$ of radius $R$ as follows: Let $S$ be a non-empty set. A metric on $S$ is a function $d: S^{2} \rightarrow \Re$ such that (a) for all $x, y \in S, d(x, y) \geq 0$; and $d(x, y)=0$ iff $x=y$; (b) $d(x, y)=d(y, x)$; and (c) $d(x, y) \leq d(x, z)+d(z, y)$. Then, for every point $p$ in $S$, there is a least number $r$ such that for all $q \in S, d(p, q) \leq r$. Call this the radius of space $S$ (from point $p$ ). The point in $S$ whose associated radius is the smallest $(r=R)$, is the center of $S$. If the space is Euclidean and finite, as is the case with Poincaré's sphere, that point is unique. ${ }^{5}$ Given this definition, if $S$ is metrically infinite, it follows immediately that no point in $S$ is the center of $S$. Hence, the definite description "the center of space", which denotes a single point in the spherical space of Poincaré's example, denotes infinitely many points in an infinite space.

The defectiveness of the notion of 'the center of space' is not of the sort one frequently finds in ambiguous ordinary phrases such as "the person I spoke with yesterday" or "the bank". In these cases, ambiguity can be avoided by further specification, such as "the person I spoke with yesterday whose name is so-and-so" and "by bank I mean 'financial institution", and so forth. Nothing of this sort can prevent "the center of space" in an infinite space from being non-unique: it is a theorem of Euclidean geometry that (infinite) space has no center. Hence, however the notion is translated into Bolyai-Lobachevsky geometry, the uniqueness condition of the definite description will not be satisfied. Even when the two theories of Poincaré's example are formulated in English, intertranslation fails in the sense that the two 
theories cannot be rendered logically equivalent. The roles played by the notion of 'center of space' in the two theories are not analogous. In fact, one might even say that it plays no role at all in Euclidean geometry. At best it can be used in theorems stating that there is no center of space, and other theorems which entail its nonexistence. Hence, the two theories, despite their empirical equivalence, remain rivals.

Similar examples of empirically equivalent but partially non-intertranslatable theories are not hard to devise. Van Fraassen (1980, pp. 49-50), for example, suggests a Newtonian theory according to which the universe rests motionless in absolute space, and a modified Newtonian theory according to which the whole universe moves in absolute space at velocity $w>0$. His example, however, is not wholly satisfactory: the two theories clearly and trivially can be rendered equivalent through translation, or reconstrual of predicates. What one theory calls "absolute velocity = 0 " is just what the other calls "absolute velocity $=w$ ".

We can, nevertheless, think of a similar example that might work as an illustration of underdetemination. In a Leibnizian physics, the notions of absolute motion and absolute space are eliminated; all motion is relative to some particular set of objects or other. The notion of absolute velocity of Newtonian physics must therefore remain untranslatable into the language of a Leibnizian physics in which motion is by definition a relational notion. But unlike the case of 'the center of space' discussed above, the translation of 'absolute motion' into the Leibnizian theory will result not in an ambiguous term, but in a term that does not denote at all. In this respect, it is very much like the notions of 'phlogiston' or 'entelechy' in relation to contemporary chemistry, for example.

Additional examples can also be summoned. Perhaps a particularly interesting one lies in the various interpretations of quantum mechanics and the debates they have spurred, which indicate that notions that have a central role in one side of the debate fail to denote or are ambiguous in the other. Regardless of what those debates entail, the point to be made here is just that some fundamental notions elude all attempts at intertranslation in the sense that the sentences in which they occur cannot be systematically rendered logically equivalent: there are always some sentences containing those notions which are true in one theory or interpretation and false in another.

Rivalry among empirically equivalent theories can thus be thought to be in principle possible, so long as intertranslation fails to render them logically equivalent. Quine's formulation of underdetermination thus already contains a sketch of what rivalry among empirical equivalent global theories should look like. As we shall see below, divergent scientific and cultural developments have brought about theories and even ordinary-language phrases and terms which resemble what Quine had in mind in that they, too, cannot be rendered logically equivalent through translation. 
There is no reason to think something similar could not happen at the level of global scientific theories; hence, the historical examples may aid the understanding of the thesis of underdetermination and offer evidence against the objection that all empirically equivalent theories should in principle be logically equivalent under some manual of intertranslation. As stated above, the examples mentioned here are no more than illustrations, since the theories are either fictitious or not examples of global theories.

\section{Translation and Commensurability}

Cases of untranslatability have been much discussed in the recent literature under the rubric of "incommensurability". A thesis of incommensurability was put forth by Kuhn (1970 [1962]) and Feyerabend (1962), which says that there are theories which fail at attempts of intertranslation: some fundamental terms and phrases of those theories might remain always foreign to its rivals. As such, the thesis of incommensurability is akin to the thesis of underdetermination, which also came to be formulated in terms of translation by Quine. What follows in this section is a comparative analysis of the two theses and of some objections that were brought against them.

The notion of incommensurability was notoriously introduced into the philosophy of science by Kuhn and Feyerabend. They did so in analogy to mathematical incommensurability: "The hypotenuse of an isosceles right triangle is incommensurable with its side or the circumference of a circle with its radius in the sense that there is no unit of length contained without residue an integral number of times in each member of the pair." (Kuhn 2000, p. 35) By analogy, theories are incommensurable if there is some term or phrase of one theory which cannot be translated into the language of the other. Such theories may even share most of their languages; incommensurability requires only that some terms and phrases are not shared. The examples from Poincaré discussed above provide an instance.

The notion of incommensurability was widely interpreted in the secondary literature as entailing the incomparability of theories. This was explicitly rejected by Kuhn. Examples of incommensurability often mentioned include the notion of 'phlogiston', which has no translation into the language of contemporary chemistry. The theories themselves, that is, phlogiston theory and contemporary chemistry can nonetheless be compared on the basis of the predictions of observations they make. The fact that some terms of one theory have no place in the other - that the sentences in which they occur have no logical equivalents in the rival theory - does not imply that the theories as wholes cannot be compared on the basis of the observation categoricals that each implies. Other examples brought up by Kuhn are the 
Newtonian notions of 'force' and 'mass', which have no counterparts in the language of Aristotelian physics, and the notion of a 'neutrino', which has no counterpart in nineteenth century English.

Quine and Kuhn came to characterize their theses of underdetermination and incommensurability in somewhat parallel ways. Both began with vaguer characterizations of the differences among rival theories, and gradually shifted towards their being partially non-intertranslatable. As already seen (section 2), Quine began his descriptions of underdetermined theories talking of alternative and conflicting empirically equivalent theories. Later he described them as logically incompatible. Finally, he settled for partial non-intertranslatability. Kuhn, of course, does not have in mind empirically equivalent theories, and the theories he describes are not global either. Nonetheless, he, too, came to think of fundamental conflicts among theories in terms of untranslatability. Incommensurable theories are rivals in the sense that the sentences of one theory contain some fundamental expressions which cannot be systematically translated into sentences of the other theory without yielding absurdities. Hence, the conflicts between empirically equivalent theories that Quine describes with his thesis of underdetermination can be seen as cases of the type of conflict Kuhn described with his thesis of incommensurability. The conflicts Quine has in mind are limited to empirically equivalent global theories, whereas the conflicts described by Kuhn extend also to other theories. Not surprisingly, both theses faced similar objections: the conflicts they describe were thought to be unintelligible and their theses were thought to be incompatible with the scientific realism defended by both authors.

In Kuhn's first writings on the topic, in particular in The Structure of Scientific Revolutions (1970a), incommensurability is presented as a relation between scientific paradigms. These, he wrote, are "universally recognized scientific achievements that for a time provide model problems and solutions to a community of practitioners" (p. viii). Incommensurability would then occur as a result of paradigm changes, or during those changes. In such periods, the "proponents of competing paradigms are always slightly at cross-purposes. Neither side will grant all the non-empirical assumptions that the other needs in order to make its case." (p. 148) Disagreements emerge not only on particular claims about nature, but also on the standards for assessing conflicting claims and on the definitions of fundamental terms:

Within the new paradigm, old terms, concepts, and experiments fall into new relationships one with the other. The inevitable result is what we must call, though the term is not quite right, a misunderstanding between the two competing schools. The laymen who scoffed at Einstein's general theory of relativity because space could not be "curved" (...) were not simply wrong or mistaken. Nor were the mathematicians, physicists, and philosophers who tried to develop a Euclidean version of Einstein's theory. What had pre-

Principia 16(1): 121-146 (2012). 
viously been meant by space was necessarily flat, homogeneous, isotropic, and unaffected by the presence of matter. (Kuhn 1970a, p. 149)

In what may have been an unjustified exaggeration, Kuhn further described those disagreements as differences in world-views: "the proponents of competing paradigms practice their trades in different worlds" (p. 150). But the main point here is that fundamental conceptual divergencies affect not only the theoretical claims but also how observable objects are perceived. Even what counts as evidence or data can be affected by which particular paradigm is adopted. The ontology of the proponents of rival theories may differ significantly, even if assent and dissent to observation sentences and categoricals remains largely unscathed by paradigm changes.

Howard Sankey (1993) has identified three phases in the development of Kuhn's thoughts on incommensurability. The first phase is the one just mentioned, which dates back to the first edition of The Structure of Scientific Revolutions (1962). It characterizes incommensurability as (i) a relation between paradigms, (ii) the outcome of changes in conceptual apparatuses which partly affect observations, and (iii) as entailing divergent world-views. The second phase occurred the 1970s, beginning with a Postscript that was added to the second edition (1970a) of Structure. During this period, Kuhn thought that comparisons between competing theories required merely that both be formulated in a single, neutral language (1970b), which would not obtain in the case of competing paradigms: "There is no neutral language into which both of the theories as well as the relevant data may be translated for purposes of comparison." (1979, p. 204) As a result, no exact translation of some fundamental notions would be possible between the two theories. This should not be taken as entailing incomparability, however:

\begin{abstract}
Most readers of my text have supposed that when I spoke of theories as incommensurable, I meant that they could not be compared. But 'incommensurability' is a term borrowed from mathematics, and it there has no such implication. The hypotenuse of an isosceles triangle is incommensurable with its side, but the two can be compared to any required degree of precision. What is lacking is not comparability, but a unit of length in terms of which both can be measured directly and exactly. In applying the term 'incommensurability' to theories, I had intended only to insist that there was no common language within which both could be fully expressed and which could therefore be used in a point-by-point comparison between them. (Kuhn 1976, p. 189)
\end{abstract}

Despite Kuhn's disclaimer, passages such as these have raised concerns among commentators. It says that although a comparison is possible, no point-by-point comparison is possible; but unless some clarification is given as to what that means, one is left with the suspicion of incoherence: a comparison is possible and is not 
possible. Kuhn's claim can be, however, more charitably interpreted as merely saying that because some fundamental terms and phrases of each rival theory cannot be translated into the language of another, there might not be any one particular theoretical sentence that one theory asserts and the other denies; hence, no 'pointby-point' comparison. A point-by-point comparison would require that divergencies be narrowed down to the truth values each theory assigns to particular sentences. Such comparison, however, will not be possible whenever divergencies between theories hinge on terms and phrases that are not intertranslatable, as may happen when theoretical sentences are considered. Some theoretical sentences which are then true in one theory will fail to have a counterpart in rival theories. Nonetheless, there will be agreement on a good portion of the terms and phrases of each theory, particularly the ones more directly linked to observation. Comparisons of the predictions of observations of each theory are thus not affected by theoretical divergencies which hinge on terms not directly fixed by observations. The notions of 'phlogiston', 'neutrino', and 'center of space' are of that nature. Comparisons of what the theories that employ those notions predict can be, however, stated without those terms. Rather, all that is required are observation sentences and categoricals. Thus, even if not all statements of each theory lend themselves to sentence by sentence or point-by-point comparison, comparison in a broader sense is still attainable.

Kuhn delivered his views in this intermediate period in a somewhat tortuous manner, perhaps due to the changes they were undergoing. He describes the comparison between incommensurable theories as made possible by the identification of the referents of each theory, which presumably should be the same. But he also seems to suggest that what counts as the referents of each theory will vary according to the theoretical assumptions each makes. At one point, he compares his thesis of incommensurability with Quine's thesis of indeterminacy of translation (see Kuhn 1970b, pp. 164-6). Clearly, however, the two theses are very different, since Quine's thesis says that for any language, various manuals of translation are possible that conform to all observable speech behavior of the speakers, whereas Kuhn's thesis says that some sentences of a theory are not translatable into the language of other theories. Kuhn's is thus a thesis that asserts untranslatability or failure of exact translation of some fundamental notions, not a plurality of alternative translations. ${ }^{6}$ The association between incommensurability and indeterminacy of translation was later dismissed by Kuhn himself (see his 1976, p. 189).

Kuhn's mature writings state what he then calls 'local incommensurability': failure to translate between localized clusters of interdefined terms. Local incommensurability, he says, is often occasioned by change or discrepancies in taxonomic categories. One of Kuhn's favorite examples of such discrepancies is the case of phlogiston theory. Although we can understand what users of that theory were attempting to say with the word "phlogiston" and describe the way they used it, neither the 
term itself nor any other can play a similar role in contemporary chemistry: "phlogiston", we now think, does not denote. We know, for example, that according to phlogiston theory, phlogiston is "given off in combustion; it reduces the elasticity and life-supporting properties of air; and so on" (Kuhn 1983, p. 43). But this description of how "phlogiston" was used does not allow for a sentence by sentence translation of phlogiston theory into contemporary chemistry. The observation categoricals that phlogiston theory and contemporary chemistry entail are not the same. Yet even if we managed to isolate some observation categoricals that are entailed by both, the theoretical principles that in each theory entails those categoricals would remain non-intertranslatable: no sentence by sentence translation can render them logically equivalent. In any such translation there are always sentences which are true in one theory and false in the other. The world, according to those theories, is governed by different laws and parceled up in different ways. Thus, even though all observation sentences and categoricals and even some theoretical statements can be rendered equivalent through translation, others cannot. And in that sense, the two theories remain partially non-intertranslatable.

In essays published in the 1980s about related issues, Putnam and Davidson objected to the characterization of incommensurability in terms of partial untranslatability. They suggested that the idea of an untranslatable theory is incoherent or unintelligible. Putnam, for example, wrote that

... if Feyerabend (and Kuhn ...) were right, then members of other cultures, including seventeenth-century scientists, would be conceptualizable by us only as animals producing responses to stimuli (including noises that curiously resemble English or Italian). To tell us that Galileo had 'incommensurable' notions and then to go on to describe them at length is totally incoherent. (1981, pp. 114-5)

According to Putnam, by describing older theories historians of science would be providing us not only with means of comparing old theories with contemporary ones but also with translations of those older theories into contemporary vocabulary. Thus, to describe what is meant by an untranslatable sentence would be incoherent: in doing so one thereby translates the sentence that is supposed to be untranslatable. Furthermore, Putnam argues, translation allows for comparison. His argument has the structure of a reductio: If the theories we held in the past and later rejected were incomparable to the ones which succeeded them, then in the history of science theory change would be an irrational affair. Each new theory would be about a wholly new set of entities, unrelated to the things referred to by the older theories. This, he argues, is implausible, since new theories are presented as better descriptions of the same objects and phenomena described by older theories. What succeeding theories refer to must largely coincide with what older theories refer to, even if they do not use the same nomenclature. ${ }^{7}$

Principia 16(1): 121-146 (2012). 
In a related discussion, Davidson says of Kuhn's cases of incommensurability that "examples like these, impressive as they occasionally are, are not so extreme". Instead, Davidson points out that "the changes and the contrasts can be explained and described using the equipment of a single language." (1984, p. 184) In particular, he says, "Kuhn is brilliant at saying what things were like before the revolution using — what else? - our post-revolutionary idiom." (ibid) Davidson's overall argument conceives the understanding of an alien point of view as based on the translation of sentences which express that point of view into our own language. This is exactly what Kuhn and other historians of science would have been doing all along. But then, Davidson argues, the very idea of an incommensurable or untranslatable sentence would be itself incoherent and unintelligible. If we cannot in principle translate a sentence or expect to see it translated in the future, why should we take it to be a sentence? Why not just dismiss it altogether as gibberish, nonsense?

These criticisms by Putnam and Davidson of the idea of incommensurability are somewhat parallel to Dummett's rejection of Quine's notion of empirically equivalent yet rival theories. Both are criticisms of the intelligibility of the idea of an untranslatable set of sentences. Putnam and Davidson, however, raise more general concerns, which affect not only empirically equivalent theories, but any two theories or languages that are allegedly untranslatable.

Quine's mitigated version of the thesis of underdetermination already suffices to set aside criticisms such as Dummett's, since Quine's thesis of underdetermination says merely that even if empirically equivalent theories can in fact be rendered logically equivalent through translation, we may nevertheless forever remain ignorant of an appropriate manual of translation. The theories would then remain rivals to us, and present alternative descriptions of the world that are equally warranted by observations. Rivalry would exist insofar as the theories posit sets of theoretical entities or principles which we cannot show to be logically equivalent by any translation manual, however devious. Whether the theoretical entities posited by each theory are actually different could, however, in principle remain forever unknown. The thesis of underdetermination, on Quine's mitigated version, says merely that there are empirically equivalent global theories which, if we find them, we will fail to render logically equivalent through translation.

Responses by Kuhn and Feyerabend take on a different strategy. They, of course, cannot concede that the examples they have in mind might in principle be intertranslatable. There is no reason to expect that, since they are not cases of empirically equivalent theories. Instead, they reject the objections by Putnam and Davidson by arguing that the cases of incommensurability that they have brought up are in fact cases of partial, or local, untranslatability. The strategy pursued by Kuhn and Feyerabend is to challenge the premises of the arguments by Davidson and Putnam. ${ }^{8}$

Putnam and Davidson assume that the thesis of incommensurability says that 
sentences from a given theory may not in general be translatable into our language. The thesis proposed by Kuhn and Feyerabend, on the other hand, says merely that sentences of a given theory may not be translatable into the language of another theory. Sentences containing "phlogiston", for example, cannot be translated into the language of contemporary chemistry. "Dephlogisticated air" may in some cases be explained as denoting, in Phlogiston Theory, the same things that in contemporary chemistry is denoted by "Oxygen" or "oxygen-rich air". However, there is no term or phrase in contemporary chemistry which could play the role of a counterpart of "phlogiston". In other words, the term "phlogiston" in Phlogiston theory fails to denote, according to contemporary chemistry. Partial translation is possible, but there is no sentence or set of sentences which can be regarded as playing the same role in both theories. This is what Kuhn called "local incommensurability", the moderate version of the thesis which he came to adopt in later writings:

Most of the terms common to the two theories function the same way in both; their meanings, whatever those may be, are preserved; their translation is homophonic. Only for a small subgroup of (usually interdefined) terms and for sentences containing them do problems of translatability arise. (Kuhn 1983, p. 36)

Feyerabend makes a similar claim: "the conditions of concept formation in one theory forbid the formation of the basic concepts of the other" (1978, p. 69). The thesis therefore does not exclude the translations that Putnam and Davidson have in mind, which are not translations of the vocabulary and sentences of one theory into the language of another, but rather from the vocabulary and sentences of one theory into the overall language we currently use and any additions that may be deemed appropriate. This is how Putnam explicitly characterizes the thesis of incommensurability:

The incommensurability thesis is the thesis that terms used in another culture, say, the term 'temperature' as used by a seventeenth-century scientist, cannot be equated in meaning or reference with any terms or expressions we possess. (Putnam 1981, p. 114)

It is also clear that translation into our overall current language is what Davidson has in mind: his discussion is not focused on translation between the languages of any two given theories but on translation into our overall language (see his 1984, pp. 190-1).

Hence, these specific criticisms by Putnam and Davidson are not criticisms of the thesis of incommensurability as it is understood by Kuhn and Feyerabend, but of a stronger thesis. The weaker thesis, dubbed "local incommensurability" by Kuhn, however, remains unaffected by those criticisms. Even if we can fully translate the 
vocabulary and the sentences of, say, Phlogiston theory, it remains true that that vocabulary and the sentences containing it cannot be fully rendered in the vocabulary and sentences of, say, contemporary chemistry. The very notion of 'phlogiston' makes no sense within contemporary chemistry. It is much like metaphysical terms such as 'entelechy' or 'grace'. We can understand how they were used in past theories and to what they intended to refer, but nothing similar or analogous can be stated without loss using only the limited technical vocabulary of contemporary chemistry. Even if Phlogiston theory and contemporary chemistry were empirically equivalent, there is no reason to expect that the sentences of one could be rendered logically equivalent to the sentences of the other. The theoretical entities and principles of each differ, and it is implausible to think that there is in this case a manual of intertranslation.

Perhaps the following examples may help: imagine a language without any temporal indexicals, say, the language of angels according to Christian theology. There are things we can say using indexicals which cannot be translated into that angelical language. For example, we can say things like "Thank goodness the war is over!" A translation of this sentence into an atemporal language might be attempted with the aid of a fixed time coordinate system: "Thank goodness the war ended at 4:30 P.M. on 2 May 2011". Both the original sentence and the translation might indeed refer to the same moment in time. But the original sentence says something which is inevitably lost in translation: it says that the war is over, that is, that the war is no longer happening. The fact that the war is now past was lost in translation. The fixed time-date coordinate does not convey that information. Of course, we can look at our watches and calendars and figure that out. But this is additional information, which we are adding to the translated sentence: "Thank goodness the war ended at 4:30 P.M. on 2 May 2011 and it is now past 4:30 P.M. on 2 May 2011". This addition, however, reintroduces the temporal indexicals which the translation was supposed to eliminate. The distinction between past, present, and future cannot be conveyed by a date-time coordinate system which does not specify the present moment. ${ }^{9}$ Losses in translation of this kind are similar to the ones which happen in the cases discussed by Kuhn and Feyerabend. Information about the intended referents can be conveyed in a language that does not contain the corresponding terms, but translation may leave out some of the information conveyed by the original sentence.

A second assumption of the arguments by Putnam and Davidson that is rejected by Kuhn and Feyerabend says that in order to understand a foreign term or language, or a rival theory, one must be able to translate it into our own language. Davidson, for example, says that

On the one hand, it is clear that speech requires a multitude of finely discriminated intentions and beliefs. (...) On the other hand, it seems unlikely that we can intelligibly attribute attitudes as complex as these to a speaker unless we can translate his words into ours. There can be no doubt that 
the relation between being able to translate someone's language and being able to describe his attitudes is very close. (Davidson 1984, p. 186; see also Putnam 1981, pp. 114-5)

Both Kuhn and Feyerabend reject this assumption. They point out that understanding can also be attained by learning the foreign language without translating it: "We can learn a language or culture from scratch, as a child learns them, without detour through our native tongue" (Feyerabend 1987, p. 76). Becoming bilingual, however, does not entail being able to translate everything one can understand in one language into the other language. Some terms and phrases can and often do remain untranslated. This is something most bilinguals experience to some extent. In the process of learning a new language, some terms and phrases will be learned in the context of a new culture. When that culture comprises practices and habits that have no counterparts in one's native culture, the newly learned terms and phrases may also fail to have counterparts or analogues in the native tongue. Translation in such cases inevitably fails, or is at best partial. ${ }^{10}$

Sometimes, in ordinary speech translation, one opts to enrich the native tongue by adding foreign terms to it. Most if not all modern languages have incorporated foreign terms to their vocabularies. "Hubris", "noir", and "Gemütlichkeit" are examples of foreign terms used in contemporary English. New phrases and terms are often added without clear restrictions on their use. In the context of a theory, however, there are more or less clear limitations on what can be added. Additions must not contradict whatever is implied by the theory, and must conform to the overall maxims of simplicity and economy in theory construction. Both these reasons preclude the notion of 'phlogiston' and others from being added to the vocabulary of contemporary chemistry. There is in general no reason to add a term which plays no role and which is independent from the rest of the theory.

We can nonetheless understand terms and phrases which are translatable into the language of a particular branch of contemporary science or all branches taken collectively. Understanding can occur by acquiring some knowledge of how it was used in past theories, which roles it played, to what it was intended to refer, etc. Of course, this is part of the process of learning the specific language to which that term belongs. This might be attained with or without translation. We might want to say that from the perspective of our overall language, that is, the technical vocabulary of the sciences plus ordinary speech, terms like "phlogiston" have been translated by historians. Perhaps we might even want to say that "phlogiston" has never ceased to be a term of the overall English language. Or we might want to say that it was lost and later recovered by the work of historians. In the latter case, "phlogiston" would now be like a foreign term (say, "hubris") which is added to the native vocabulary in the overall process of translation. Whichever of these alternatives is the case, the 
thesis of incommensurability or "local incommensurability" still stands: "phlogiston" and sentences from Phlogiston theory which contain it cannot be translated without loss into the technical language of contemporary chemistry.

Kitcher (1978, pp. 534-5) has persuasively argued that the language of contemporary chemistry can be used to find out what the intended referents of the phrase "dephlogisticated air" was: in some contexts it was used to refer to oxygen while in other contexts it had no referent. This shows that we can describe how the phrase was used and by doing so come to understand it. But this still gives us no translation of the term into the language of contemporary chemistry. Phlogiston theory posited the existence of a single "principle", phlogiston, which we can now identify as absence of oxygen in the air in some cases and as nothing at all in other cases. Such attempts at translation thus forcibly eliminate the claim made by the Phlogiston theory that there would be a single principle in all such instances. That idea is lost in translations of the term into the language of contemporary chemistry. The ontology of Phlogiston theory cannot be mapped onto the ontology of contemporary chemistry.

The knowledge we may acquire of the use of a term such as "phlogiston" or of any other term from theories we no longer adopt is similar to the learning of a culture which is foreign to our own. We gradually become aware of the circumstances in which a term is correctly used in that theory or culture, but may remain incapable of using anything similar in our own theory or culture. We can come to describe how it was or is used but may remain unable or unwilling to use it in describing the world from our own perspective. In finding out how such terms were used we in fact acquire the technical vocabulary of a foreign language. One may, in special circumstances, come to use those terms in the first person present indicative tense. But insofar as we know that the theories to which they belong are false, such circumstances must remain extraordinary. The terms learned relate to each other in ways that may have no parallel in our native tongue.

Kuhn illustrates the point with ordinary language examples. He distinguishes two kinds of difficulties in the translation of foreign phrases. Some phrases may be ambiguous, and the translator must vary his English rendering of the phrase according to the contexts in which it is originally used. The French word "pompe" is a good example: "In some contexts (typically those involving ceremonies), its English equivalent is 'pomp'; in other contexts (typically hydraulic), its equivalent is 'pump'." (Kuhn 1983, p. 48) In contrast, some words have no clear-cut equivalents in English, even when context of use is taken into consideration. In their translations into English, something gets lost. The net of interrelated notions which they allude to has no counterpart in the language into which it is to be translated:

... contrast the case of 'pompe' with that of French words like 'esprit' or

Principia 16(1): 121-146 (2012). 
'doux'/'douce'. 'Esprit' can be replaced, depending on context, by such English terms as 'spirit', 'aptitude', 'mind', 'intelligence', 'judgment', 'wit', or 'attitude'. The latter, an adjective, can be applied, inter alia, to honey ('sweet'), to wool ('soft'), to underseasoned soup ('bland'), to a memory ('tender'), or to a slope or a wind ('gentle'). These are not cases of ambiguity, but of conceptual disparity between French and English. Esprit and doux/douce are unitary concepts for French speakers, and English speakers as a group possess no equivalents.(Kuhn 1983, p. 48)

These examples help us understand why poetry is so difficult to translate. Poetry relies on spontaneous associations and allusions which the mere utterance of a word brings to the native speaker. Those associations and allusions may be wildly different in foreign languages. For that reason, one can almost define poetry as that which is lost in translation. Likewise, humor is frequently lost, and for the same reason.

A similar case, from inter-cultural studies, is brought up by MacIntyre, in a discussion of Confucian and Aristotelian virtue theories:

... while both the Confucian and the Aristotelian moralist will see and report one and the same person giving freely and liberally to someone else in need, the Confucian may observe an absence of $l i$, of that ritual formality which is an essential characteristic of jen, a type of absence necessarily invisible to the Aristotelian, who has no words in either Aristotle's Greek or William of Moerbeke's Latin to translate li, an expression captured neither by such words as ethos, hostia, orgia, teletai used of religious rituals, nor by such words as ethos, signifying the customary and habitual, nor by their medieval Latin equivalents. By contrast, the Aristotelian will observe (...) an example of a disposition as evidencing a particular ordering or disorder of the psyche, a conformity or lack of it to what is required of a citizen of the polis, both understood in terms of an ultimate telos conceived in a highly specific way, all of which must be invisible to the Confucian who has no words for psyche or polis either in the ancient Chinese of Confucius or in the later Chinese of Sung Neo-Confucianism. (MacIntyre 1991, p. 110)

The point of these examples is that the roles played by particular notions in theories and in cultures may have no counterpart in other theories and cultures. Thus, at best one can describe its role but not adequately translate it and use it within a foreign culture. One can add the foreign word to the native vocabulary. But if it is to retain the same role it had in the foreign culture, it is bound to remain partially untranslatable from the standpoint of our own culture, in the sense that we can describe its use, but not actually use it to describe the world.

These examples from cultural studies are strictly analogous to the ones from the history of science discussed by Kuhn and Feyerabend. A set of interrelated notions that is used in one theory or culture has no place or counterpart in another. In each case one can come to understand how they were used and accurately describe their 
correct uses, but cannot put oneself to use those notions while maintaining one's own theory of the world or without giving up part of one's native culture. These examples also lend additional credibility to Quine's thesis of underdetermination. They provide illustrations of ways in which interrelated sets of sentences from a particular theory or culture may elude all attempts to render logically equivalent to sets of statements in rival theories or foreign cultures. Additionally, they show how divergent cultural and scientific developments can produce such sentences.

\section{Indeterminacy of translation and underdetermination}

In a story by Borges, the philosopher Averroës finds himself at a loss in the translation of a couple of words in Aristotle's Poetics: "Those words were 'tragedy' and 'comedy'. He had come across them years earlier, in the third book of the Rhetoric; no one in all of Islam could hazard a guess as to their meaning." (Averroës' Search, p. 70) What Averroës lacks, in Borges's story at least, is not so much an adequate supply of words but the corresponding culture in which the theatrical practices of tragedy and comedy had their place. In the absence of such contexts, the two words would remain not only untranslatable, but also largely incomprehensible for him. These cases of untranslatability are to be contrasted with the possibilities allowed for by Quine's thesis of indeterminacy of translation.

The thesis of indeterminacy says that "manuals for translating one language into another can be set up in divergent ways, all compatible with the totality of speech dispositions, yet incompatible with one another" (Quine 1960, p. 27). The cases we have been discussing so far are not cases of indeterminacy of translation, but rather of partial untranslatability. Indeterminacy says that where one translation manual is possible at all, various others, incompatible with it, are also possible. ${ }^{11}$

Quine distinguishes two theses of indeterminacy: the indeterminacy of sentences (or "holophrastic indeterminacy") and the indeterminacy of reference (or "ontological relativity"). The former, he says, is a conjecture, whereas the latter admits a trivial proof:

Woods misinterprets me as saying that the indeterminacy of holophrastic meaning is more serious than that of reference. I would say the opposite. My point was just that the former is conjectural and does not follow from the latter, which admits of trivial proof. The essence of the proof is just that $x$ is an $F$ if and only if the proxy of $x$ is the proxy of $F$. (Quine 1998a, p. 728)

Both versions of the thesis make an ontological claim. They are not theses about what can or cannot be known by observation of a foreign speech behavior. ${ }^{12}$ Rather, the point is precisely that beyond observable speech behavior there is nothing else 
to be known: "There is nothing in linguistic meaning beyond what is to be gleaned from overt behavior in observable circumstances" (Quine 1992b, p. 38). Hence, in a theory or system of the world, there is no need to posit the existence of meanings.

The correctness of translation is measured by the fluency of communication and negotiation for which it allows. In principle, alternative manuals can work equally well in that regard. They can be incompatible yet correct insofar as they allow for equally fluent communication across languages. The thesis applies not only to the translation of foreign languages but also to translations of the native speech (Quine 1992b, p. 48). ${ }^{13}$ What it shows is that ontology is relative to a manual of translation, and that "the notion of propositions as sentence meanings is untenable" (Quine 1992b, p. 102).

The thesis of underdetermination, on the other hand, makes no claims about ontology; rather, its point is epistemological: alternative descriptions of the world can be equally warranted. Hence, the thesis of indeterminacy would obtain even if the thesis of underdetermination were false. Even if observations determined one particular theory of the world, translation would still be indeterminate. Observable speech behavior is compatible with divergent manuals of translations, even if observations determined one particular physical theory of the world. What remains indeterminate are the notions of traditional semantics, in particular the notion of 'meaning'.

The indeterminacy of translation differs from the underdetermination of science in that there is only the natives' verbal behavior for the manuals of translation to be right or wrong about; no claims are laid regarding hidden neural mechanisms. If translators disagree on the translation of a Jungle sentence but no behavior on the part of the Jungle people could bear on the disagreement, then there is simply no fact of the matter. In the case of natural science, on the other hand, there is a fact of the matter, even if all possible observations are insufficient to reveal it uniquely. The facts of nature outrun our theories as well as all possible observations, whereas the traditional semantics outruns the facts of language. (Quine 1987, pp. 9-10)

Some authors have questioned the compatibility of the thesis of indeterminacy of translation with the thesis of underdetermination. ${ }^{14}$ Underdetermination entails rivalry between empirically equivalent theories, but empirically equivalent theories, given indeterminacy of translation, can be rendered compatible. According to Quine's formulation of the thesis of in the late 1960s and early 1970s, underdetermination should in fact entail the possibility of empirically equivalent yet logically incompatible theories. That version of the thesis might indeed bring about irresoluble problems for Quine's thesis of indeterminacy of translation, as some have argued.

However, when underdetermination is conceived as entailing empirically equivalent but partially untranslatable theory formulations, the inconsistency dissipates 
altogether. The fact that there is more than one correct manual of translation shows that there are more translations than one might have thought; but that does not show that there is a translation between every pair of empirically equivalent theories. The thesis of indeterminacy and the modest thesis of underdetermination - as Quine understood it from 1975 onwards - make different but compatible claims. The first says that the correctness of translation rests solely on the fluency of communication for which it allows, and not on some ulterior notion of meaning. The thesis of indeterminacy asserts that ontology is relative to a translation manual, and that the notion of propositions as sentence meanings is untenable. Additionally, the thesis thus recommends ontological simplification: the traditional notions of meaning and proposition might be altogether eliminated from a regimented theory of the world. The thesis of underdetermination, on the other hand, makes an epistemological claim: alternative, mutually untranslatable theories of the world can be equally warranted by observations.

\section{Acknowledgements}

This is a revised version of chapter 4 of my $\mathrm{PhD}$ dissertation. For comments and discussion, I would like to thank Peter Hylton, Daniel Sutherland, Nick Huggett, Carl Hoefer, David Harker, Dearbhla Kelly, Andrew Blom, Rogério Saucedo Corrêa, Paulo Faria, Sílvia Altmann, and André Klaudat. Many thanks also to an anonymous referee for this journal.

\section{References}

Austin, D. 1990. What's the meaning of "this"? Ithaca, NY: Cornell University Press.

Bar-On, D. 1986. Semantic indeterminacy and scientific underdetermination. Pacific Philosophical Quarterly 67: 254-63.

Bechter, P. W. 1980. Indeterminacy and underdetermination: are Quine's two theses consistent? Philosophical Studies 38: 309-20.

Borges, J. L. 2004. Averroës' search. In: J. L. Borges, The Aleph and other stories. New York: Penguin Books.

Chisholm, R. 1982. The first person: an essay on reference and intentionality. Minneapolis: University of Minnesota Press.

Davidson, D. 1984. On the very idea of a conceptual scheme. In: D. Davidson, Inquiries into truth and interpretation. Oxford: Oxford University Press, pp. 183-98.

Dummett, M. 1981 [ $1^{\text {st }}$ ed. 1973]. Frege: philosophy of language. $2^{\text {nd }}$ ed. Cambridge, Mass.: Harvard University Press.

Earman, J. 1993. Underdetermination, realism and reason. Midwest Studies in Philosophy 18: 19-38.

Feyerabend, P. 1962. Explanation, reduction, and empiricism. Minnesota Studies in the Philosophy of Science 3: 27-97.

Principia 16(1): 121-146 (2012). 
1987. Putnam on incommensurability. British Journal for the Philosophy of Science 38: 75-81.

Friedman, M. 2002. Kant, Kuhn, and the rationality of science. Philosophy of Science 69: 171-90.

Gale, R. 1962. Tensed statements. The Philosophical Quarterly 12: 53-9.

Gibson Jr., R. F. 1998 [ $1^{\text {st }}$ ed. 1986]. Translation, physics, and facts of the matter. In: L. E. Hahn \& P. A. Schilpp (eds.) The philosophy of W. V. Quine, $2^{\text {nd }}$ exp. ed. La Salle, Ill.: Open Court, pp. 139-54.

Hartshorne, R. 2000. Geometry: Euclid and beyond. New York: Springer.

Hoefer, C. \& Rosenberg, A. 1994. Empirical equivalence, underdetermination, and systems of the world. Philosophy of Science 61: 592-607.

Kitcher, P. 1978. Theories, theorists and theoretical change. Philosophical Review 87: 519-47.

Kuhn, T. 1970a [ $1^{\text {st }}$ ed. 1962]. The structure of scientific revolutions, $2^{\text {nd }}$ ed. Chicago: University of Chicago Press.

- 2000. The road since 'Structure'. Chicago: University of Chicago Press.

Kukla, A. 1998. Studies in scientific realism. New York: Oxford University Press.

Laudan, L. \& Leplin, J. 1991. Empirical equivalence and underdetermination. Journal of Philosophy 88: 449-72.

MacIntyre, A. 1991. Incommensurability, truth, and the conversation between Confucians and Aristotelians about the virtues. In: E. Deutsch (ed.), Culture and modernity: East-West philosophic perspectives. Honolulu: University of Hawaii Press, pp. 104-22.

Martens, D. 1994. Demonstratives, descriptions, and knowledge: a critical study of three recent books. Philosophy and Phenomenological Research 54: 947-63.

Park, S. 2009. Philosophical responses to underdetermination in science. Journal for the General Philosophy of Science 40: 115-24.

Perry, J. 1979. The problem of the essential indexical. Noûs 13: 3-2-1.

Poincaré, H. 1952 [1905]. Science and hypothesis. New York: Dover.

Prior, A. 1959 Thank goodness that's over! Philosophy 34: 12-17.

Putnam, H. 1973. Explanation and reference. In: H. Putnam, (1975) Mind, language and reality: Philosophical papers, volume 2. Cambridge: Cambridge University Press, pp. 196214.

- 1981. Reason, truth and history. Cambridge: Cambridge University Press.

1984. What is realism? In: J. Leplin (ed.), Scientific realism. Berkeley: University of California Press, pp. 140-53.

Quine, W. 1955. Posits and reality. In W. V. Quine, (1976) The ways of paradox and other essays, rev. and enlarged ed. Cambridge, Mass.: Harvard University Press, pp. 246-54.

- 1960. Word and object. Cambridge, Mass.: MIT Press.

. 1969a. Epistemology Naturalized. In: W. V. Quine, Ontological relativity and other essays. New York: Columbia University Press, pp. 69-90.

. 1969b. Reply to Chomsky. In: D. Davidson \& J. Hintikka (eds.) Words and objections: essays on the work of W.V. Quine. Dordrecht: D. Reidel, pp. 302-11.

- 1970. On the reasons for indeterminacy of translation. Journal of Philosophy 67: 178-83.

—. 1975a. On empirically equivalent systems of the world. Erkenntnis 9: 313-28.

Principia 16(1): 121-146 (2012). 
1975b. The nature of natural knowledge. In: S. Guttenplan (Ed.), Mind and language.

Oxford: Oxford University Press, pp. 67-81.

. 1976. A comment on Grünbaum's claim. In: S. G. Harding (ed.) Can theories be re-

futed? Essays on the Duhem-Quine thesis (Synthese Library, vol. 81). Dordrecht: D. Reidel, p. 132.

- 1979. Comments on Newton-Smith. Analysis 39: 66-7.

1981. Theories and things. Cambridge, Mass.: Harvard University Press.

1987. Indeterminacy of translation again. Journal of Philosophy 84: 5-10.

1990a. Comment on Bergström. In: R. Barrett \& R. Gibson (eds.), Perspectives on

Quine. Cambridge, Mass.: Basil Blackwell, pp. 53-4.

— 1990b. Three indeterminacies. In: R. Barrett \& R. Gibson (eds.), Perspectives on

Quine. Cambridge, Mass.: Basil Blackwell, pp. 1-16.

- 1992a. Commensurability and the alien mind. Common Knowledge 1: 1-2.

- 1992b. Pursuit of Truth. rev. ed. Cambridge, Mass.: Harvard University Press.

- 1998a. Reply to John Woods. In: L. E. Hahn \& P. A. Schilpp (eds.), The philosophy of

W.V. Quine, $2^{\text {nd }}$ exp. ed. La Salle, Ill.: Open Court, pp. 726-28.

- 1998b. Reply to Paul A. Roth. In: L. E. Hahn \& P. A. Schilpp (eds.), The philosophy of

W.V. Quine, $2^{\text {nd }}$ exp. ed. La Salle, Ill.: Open Court, pp. 459-61.

Sankey, H. 1990. In defence of untranslatability. Australasian Journal of Philosophy 68: 1-21.

- 1993. Kuhn's changing concept of incommensurability. British Journal for the Philos-

ophy of Science 44: 759-74.

Schiffer, S. 1978. The basis of reference. Erkenntnis 13: 171-206.

Severo, R. 2008. Plausible insofar as it is intelligible: Quine on underdetermination. Synthese

161: 141-65.

Sklar, L. 1975. Methodological conservatism. The Philosophical Review 84: 374-400.

. 1981. Do unborn hypotheses have rights? Pacific Philosophical Quarterly 62: 17-29.

Stalnaker, R. 1981. Indexical belief. Synthese 49: 129-51.

Stanford, P. K. 2001. Refusing the devil's bargain: what kind of underdetermination should

we take seriously? Philosophy of Science 68: S1-S12.

Van Fraassen, B. 1980. The scientific image. Oxford: Clarendon Press.

Wisdom, J. 1974. The incommensurability thesis. Philosophical Studies 25: 299-301.

Wittgenstein, L. 1958. Philosophical investigations. Oxford: Blackwell.

Yalçin, Ü. D. 2001. Solutions and dissolutions of the underdetermination problem. Noûs 35: 394-418.

Rogério Passos SEVEro

Departamento de Filosofia

Universidade Federal de Santa Maria

Av. Roraima 1000, prédio 74-A 97105-900 Santa Maria, RS

BRASIL

rpsevero@smail.ufsm.br

Resumo. Uma das objeções à tese da subdeteminação de teorias pelas observações consiste em dizer que é ininteligível. Duas teorias empiricamente equivalentes quaisquer - diz a objeção - seriam em princípio intertraduzíveis, e por isso não poderiam ser consideradas rivais

Principia 16(1): 121-146 (2012). 
em nenhum sentido não trivial. Contra essa objeção, este artigo mostra que teorias empiricamente equivalentes podem conter frases teóricas que não são intertraduzíveis. Exemplos são extraídos de uma discussão relacionada a esta sobre incomensurabilidade, para mostrar que a não-intertraduzibilidade de frases teóricas é possível.

Palavras-chave: Equivalência empírica; subdeterminação; incomensurabilidade; intraduzibilidade.

\section{Notes}

${ }^{1}$ See Park 2009 for an alternative, more comprehensive description of the different kinds of underdetermination.

${ }^{2}$ See Laudan and Leplin 1991, Hoefer and Rosenberg 1994, Quine 1975a, and Stanford 2001.

${ }^{3}$ For a comprehensive discussion of these changes, see this author's 2008.

${ }^{4}$ On Quine's notion of an 'observation categorical', see Quine 1981, p. 27; 1992b, pp. 9-11.

${ }^{5}$ The uniqueness of the center of a sphere can be proved as follows: suppose, for reductio, that the center is not unique. Then there are two points, $p_{1}$ and $p_{2}$, whose associated radii, $r_{1}$ and $r_{2}$, are equal to each other $\left(r_{1}=r_{2}\right)$ and smaller than that of any other point in $S$ which are not equal to either of them. Since $p_{1} \neq p_{2}, d\left(p_{1}, p_{2}\right)>0$. Pick any number $a \in \Re$ such that $0<a<d\left(p_{1}, p_{2}\right)$. Since $p_{2} \in S, a<r_{1}$. So, $0<r_{1}-a<r_{1}$. So there is a $q \in S$ such that $p_{2}$ is between $p_{1}$ and $q$ and $d\left(p_{1}, q\right)=r_{1}-a$. ['Betweenness' is a relation which satisfies at least the following three axioms: (1) If $B$ is between $A$ and $C$ (written $A * B * C$ ), then $A, B, C$ are three distinct points on a line, and also $C * B * A$. (2) For any two distinct points $A, B$, there exists a point $C$ such that $A * B * C$. (3) Given three distinct points on a line, one and only one of them is between the other two. (See Hartshorne 2000, p. $73 \mathrm{ff}$.)] Since $r_{1}=r_{2}, r_{1}-a=r_{2}-a$. Since $p_{2}$ is between $p_{1}$ and $q, d\left(p_{2}, q\right)=r_{2}-a=r_{1}-a$. Given that $p_{2}$ is between $p_{1}$ and $q$, it also follows that $d\left(p_{1}, q\right)=d\left(p_{1}, p_{2}\right)+d\left(p_{2}, q\right)$. So $d\left(p_{1}, q\right)=d\left(p_{1}, p_{2}\right)+r_{1}-a$. Since $a$ was chosen such that $0<a<d\left(p_{1}, p_{2}\right)$, it follows that $d\left(p_{1}, p_{2}\right)-a>0$. Hence, $d\left(p_{1}, p_{2}\right)-a+r_{1}>r_{1}$. But $d\left(p_{1}, p_{2}\right)-a+r_{1}=d\left(p_{1}, p_{2}\right)+r_{1}-a=$ $d\left(p_{1}, q\right)$. Hence, $d\left(p_{1}, q\right)>r_{1}$. So $p_{1}$ is not a center of $S$, because $q \in S$ and $d\left(p_{1}, q\right)>r_{1}$.

${ }^{6}$ Further discussion in Sankey (1991).

${ }^{7}$ See his 1973, pp. 197 ff. A similar argument had already been put forth by Wisdom 1974.

8 The following few paragraphs draw considerably on Sankey 1990, pp. 1-11.

${ }^{9}$ Yet another attempt to get rid of the temporal indexical of the original sentence would be to say something like "Thank goodness the war ended at 4:30 P.M. on 2 May 2011 and this sentence is uttered after 4:30 P.M. on 2 May 2011". But again, if this latter sentence contains no temporal indexical, then the verb "is" cannot be understood to be in the present tense. Instead, we should read it as an atemporal "is", and in that case the resulting claim does not say whether the sentence has been uttered, is being uttered, or will be uttered. Hence, it does not say whether the war has ended in the past, is ending at this moment, or will end in the future. Furthermore, the phrase "this sentence is uttered after 4:30 P.M. on 2 May 2011", when uttered, works like any other temporal indexical, since the moment in time it refers to varies according to the context of utterance: to say "this sentence is uttered" while uttering the sentence is equivalent to say "this sentence is being uttered now". See Prior 1959, Gale 
1962, Schiffer 1978, Perry 1979, Stalnaker 1981, Chisholm 1982, Austin 1990, and Martens 1994 for further discussion.

${ }^{10}$ A similar point was suggested by Wittgenstein (1958): “... to imagine a language means to imagine a form of life" ( $\S 19)$; “... the speaking of language is part of an activity, or of a form of life" (§ 22).

${ }^{11}$ In later works, Quine explains the sense in which manuals may be considered incompatible: two manuals equally compatible with the behavior of native speakers "might not be usable in alternation, from sentence to sentence, without issuing in incoherent sequences. Or, to put it another way, the English sentences prescribed as translation of a given Jungle sentence by two rival manuals might not be interchangeable in English contexts." (Quine 1992b, p. 48)

${ }^{12}$ For an excellent discussion of this point, see Gibson 1998.

${ }^{13}$ A common objection to the thesis says that if it is true and applies to our home language, then we cannot really know what we mean by what we say. When referring to an object, do we mean that particular object or the complement of that object, that is, the whole universe minus that object? Which of various possible manuals of translation are we using while speaking our home language? Quine dismisses these objections: "Within the home language, reference is best seen (...) as unproblematic but trivial, on a par with Tarski's truth paradigm. Thus 'London' denotes London (whatever that is) and 'rabbit' denotes rabbits (whatever they are)." (Quine 1998b, p. 460)

${ }^{14}$ For example, Bechtel 1980, Bar-On 1986, Gemes 1991, and Yalçin 2001.

Principia 16(1): 121-146 (2012). 